\title{
Statistical Physics of Unzipping DNA
}

\author{
David R. Nelson \\ Lyman Laboratory of Physics, Harvard University, Cambridge, MA 02138. \\ E-mail: nelson@cmt.harvard.edu
}

(November 2, 2018)

\begin{abstract}
The denaturation of double-stranded DNA as function of force and temperature is discussed. At room temperature, sequence heterogeneity dominates the physics of single molecule force-extension curves starting about 7 piconewtons of below a $\sim 15 p N$ unzipping transition. The dynamics of the unzipping fork exhibits anomalous drift and diffusion in a similar range above this transition. Energy barriers near the transition scale as the square root of the genome size. Recent observations of jumps and plateaus in the unzipping of lambda phage DNA at constant force are consistent with these predictions.
\end{abstract}

\section{SINGLE MOLECULE BIOPHYSICS EXPERIMENTS}

The past decade has seen a revolution in biophysics, due to exquisitely sensitive experiments [1] which probe ingredients of the "central dogma" of molecular biology [2] at the level of individual molecules. Although exceptions exist, the central dogma states that biological information first flows from DNA to messenger RNA via transcription mediated by a RNA polymerase. Information is then transferred from mRNA to proteins via a ribosomemediated translation process.

Among the efforts to probe the basic constituents further: (a) Proteins anchored to a microscope slide in their biologically relevant folded state have been teased apart with atomic 
force probes [3] attached using, e.g., biotin-streptavidin linkages; (b) individual DNA's linked to magnetic beads have been stretched and twisted by small magnetic field gradients, allowing studies of both supercoiling and twist-induced denaturation [4]; and (c) the reversible unfolding of single RNA molecules (with beads attached to both ends) has been studied using laser tweezers with a feedback loop to generate a constant force $F$ [5]. In this later experiment, the fluctuating displacements of a $\sim 25$ base-pair RNA hairpin evolve from a predominantly closed state (with rare opening events) at small force to a predominantly open state (with rare closing events) at larger forces. At an intermediate force of $F_{c} \approx 14$ piconewtons $(p N)$, the hairpin spends approximately equal time in both configurations, suggestive of a first-order phase transition between the open and closed states in the "thermodynamic limit" of a very large hairpin. 


\section{FIGURES}

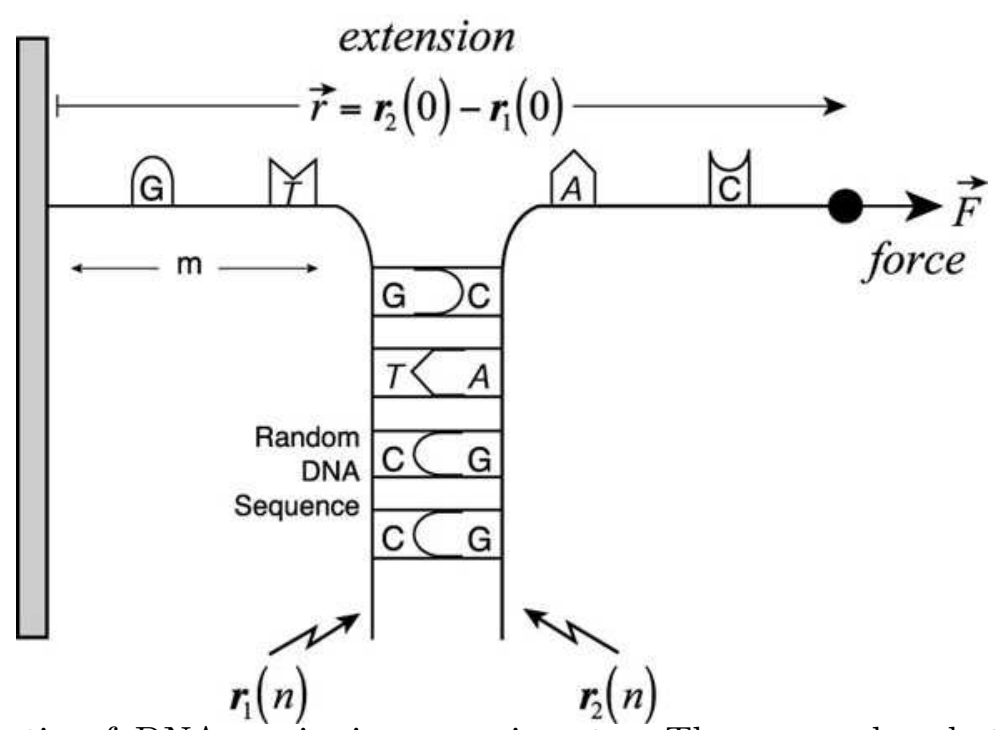

FIG. 1. Schematic of DNA unzipping experiments. The sugar-phosphate backbones, with complementary base pairing, are represented by the functions $\mathbf{r}_{1}(n)$ and $\mathbf{r}_{2}(n)$, where $n$ indexes base pairs. Experiments can be carried out either at constant extension $\mathbf{r}_{1}(0)-\mathbf{r}_{2}(0)$ or at constant force $\vec{F}$.

DNA force denaturation experiments related to the subject of this review have been carried by Bockelmann et al. [6-8] (see Fig. 1). In this pioneering work, the 48,502 base pairs of the virus phage lambda with one strand attached to a microscope slide were unzipped by a microneedle attached to the other strand under conditions which produced a constant rate of unzipping at a fixed temperature. Reasonable agreement with these experiments was obtained by direct numerical evaluation of the equilibrium statistical sums associated with a given average degree of unzipping. If the rate of unzipping is slow, such experiments effectively probe the physics at constant extension. The average force under these conditions locks in at about 14-15 piconewtons. The time-dependent fluctuations of this force as the unzipping proceeds provide information about the particular sequence of $\mathrm{G}$ :C and A:T base pairs being torn apart [6-8]. The full phase diagram of DNA in the force-temperature plane is shown in Fig. 2 [9-13]. The thermal DNA melting transition at zero force (see Sec. 2) is characterized by diverging length scales associated with denaturation bubbles. When $F \neq 0$, 
the native duplex DNA denatures into an unzipped state via a first-order phase transition (see Sec. 3). Under conditions of a slow, constant rate of extension [6-8], the average force adjusts so that the system sits on the heavy first-order transition line $F_{c}(T)$. At any stage in the unzipping process, the unzipping fork separating the native and unzipped states is like the meniscus dividing, say, liquid and gas phases at constant volume at a bulk first-order phase transition.

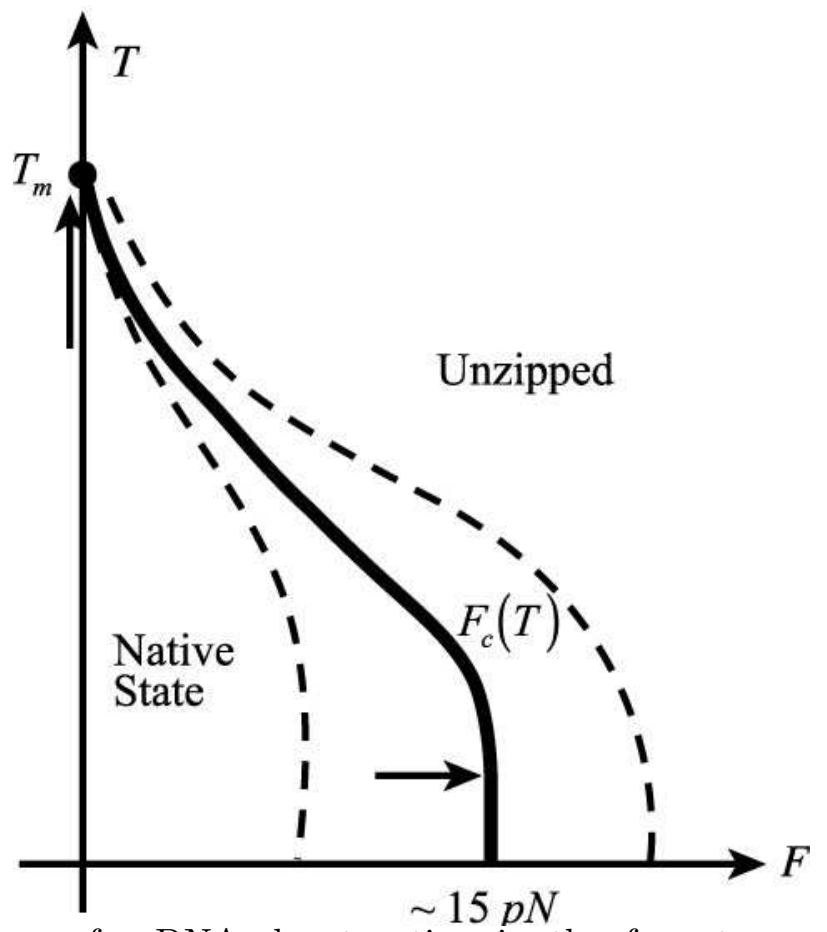

FIG. 2. Phase diagram for DNA denaturation in the force-temperature plane. Conventional thermal denaturation occurs at zero force as $T$ approaches the melting temperature $T_{m}$. Force-induced denaturation, or unzipping occurs on the path indicated by the horizontal arrow. Sequence heterogeneity dominates the physics between the two dashed crossover lines. 


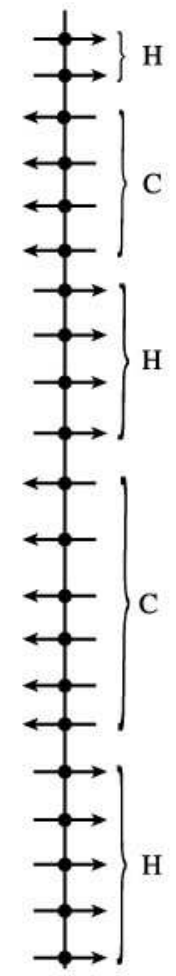

(a)

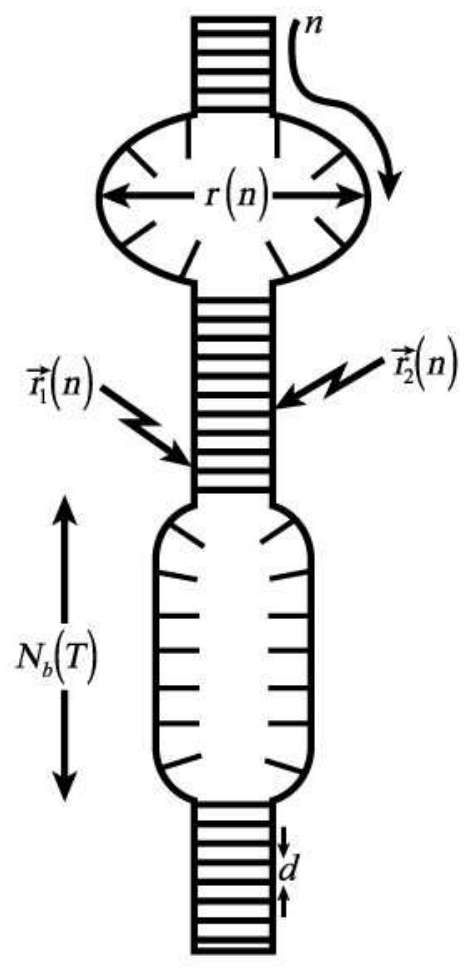

(b)

FIG. 3. Models of thermal denaturation. (a) Ising model, where right-pointing spins represent hydrogen-bonded "helix" segments, and left-pointing spins represent denatured "coil" segments. (b) More sophisticated polymer model of denaturation whose statistical mechanics is mapped onto a problem in three-dimensional quantum mechanics. $N_{b}(T)$ is the number of base pairs associated with a typical denatured "coil" segment.

Although running unzipping experiments in a two-phase region associated with the line $F_{c}(T)$ is well-suited for possible DNA sequencing applications [6-8], the full forcetemperature phase diagram of Fig. 2 is interesting for a number of reasons. First, understanding DNA denaturation under conditions of constant force (instead of constant extension) could provide insights into the complicated process by which DNA replicates during bacterial cell division [15]. Second, statistical mechanics in ensembles with intensive variables like the force held fixed is often more tractable than when the control parameter is a conjugate variable like the extension. Precise analytic predications are indeed possible in the vicinity of $F_{c}$, even when sequence disorder is present $[9,14]$. Third, experiments which use force as a control parameter can probe interesting behavior of the unzipping fork for 
$F<F_{c}(T)$ (analogous to "wetting" phenomena in bulk phase transitions [16]) and anomalous fork dynamics for $F>F_{c}(T)$. Because of significantly different bonding strengths of the nucleotide pairs A:T (two hydrogen bonds) and G:C (three hydrogen bonds), heterogeneity dominates unzipping over a large region of the phase diagram, for $\frac{1}{2} F_{c} \lesssim F \lesssim \frac{3}{2} F_{c}$, inside the region bounded by the dashed lines in Fig. 2 .

\section{THERMAL DENATURATION OF DNA}

In this Section we discuss the thermal denaturation of DNA at zero force (vertical arrow in Fig. 2). This transition plays an important role in the polymerase chain reaction (PCR), where it is applied cyclically to amplify minute amounts of DNA. The basic physical ideas driving thermal denaturation (worked out in the 1960s) are described in Refs. [17-19]. The simplest models invoke the Ising-like description of DNA shown in Fig. 3a, where right-facing spins represent bonded nucleotides in a closed "helix" state and left-facing spins the open "coil" state. The magnetic field of the equivalent Ising model represents a (temperaturedependent) nucleotide bonding free energy and the Ising exchange energy determines an "initiation factor" at the boundaries between the helix and coil regions. The statistical mechanics of this one-dimensional Ising model can be described entirely in terms of kink excitations associated with transitions between domains of up and down spins, which here represent helix/coil boundaries. Rapid variations in DNA from a helix-dominated to a coildominated state are predicted by such models [19], corresponding to the magnetic field changing sign in the equivalent Ising system. However, it is well-known that the 1d Ising model does not have a real phase transition at finite temperature, basically because the finite energy cost of kinks at zero external field is overwhelmed by the entropy gained by creating them [20]. An improved theory results when the three-dimensional entropy of wandering loops in the coil sections (see Fig. 3b) is taken into account. This can be done by approximating the loops as ideal random walks [17], or improving this estimate by taking self-avoidance of the loops into account [21]. The effect on the statistical mechanics is to 
produce a long range interaction which binds kinks together and leads to a continuous finite temperature phase transition [17-19]. Recent work by Kafri et al. incorporates additional self-avoidance between coil sections and the rest of the DNA chain (important for large molecules) and argues that the thermal denaturation transition then becomes first order $[22]$.

Heterogeneity of the bonding between base pairs ( $\mathrm{G}: \mathrm{C}$ bonds are stronger than A:T ones) is neglected in the treatments above. Simple helix-coil descriptions with heterogeneity are governed by the statistical mechanics of the $1 \mathrm{~d}$ random field Ising model. A reasonable guess for more realistic models with heterogeneity is that while the transition may be less sharp (weakly bound regions melt before more tightly bound ones), a real finite temperature phase transition survives. Lyubchenko et al. [23] have calculated numerically the effect of sequence heterogeneity on the DNA differential melting curves for the 5375 nucleotides of the virus $\phi X 174$. For a recent theory of thermal denaturation of heterogeneous DNA, see Ref. [24].

In the remainder of this section, we discuss how thermal denaturation of "homogeneous" DNA with identical base pairs can also be understood by mapping the statistical mechanics onto the delocalization of a quantum mechanical particle in three dimensions. This is the approach we will generalize in Sec. 3 to study force-induced naturation.

Referring to Fig. 3b, we parameterize the positions of the two (antiparallel) sugarphosphate backbones of DNA in three dimensions by the functions $\mathbf{r}_{1}(n)$ and $\mathbf{r}_{2}(n)$, where $n$ is an integer indexing $N$ base pairs $(0 \leq n \leq N)$. We model each strand separately as a Gaussian random coil [25] . For example, considering strand 1 in isolation, the probability of a particular polymer conformation $\mathbf{r}_{1}(n)$ is proportional to $e^{-F_{1}\left[\mathbf{r}_{1}(n)\right] / T}$ (we use units such that $k_{B}=1$ ), where

$$
F_{1}\left[\mathbf{r}_{1}(n)\right]=\frac{1}{2} K \int_{0}^{N d}\left(\frac{d \mathbf{r}_{1}(s)}{d s}\right)^{2} d s
$$

Here we have used a convenient continuum notation as shorthand for a discrete sum over $n$ and replaced $n$ by the arclength $s=n d$, where $d$ is the spacing between nucleotide monomers. 
To relate the spring constant $K$ to the single strand persistence length, use (1) to evaluate $\left\langle\left|\vec{r}_{1}(N d)-\vec{r}_{1}(0)\right|^{2}\right\rangle$, with the result

$$
\left\langle\left|\vec{r}_{1}(N d)-\vec{r}_{1}(0)\right|^{2}\right\rangle=\frac{3 T N d}{K}
$$

From the definition of persistance length $\ell$, namely $\left\langle\left|\vec{r}_{1}(N d)-\vec{r}_{1}(0)\right|^{2}\right\rangle \equiv \ell N d$, we have [26]

$$
K=\frac{3 T}{\ell}
$$

where $\ell \approx 10 \AA$ for single-stranded DNA at physiological temperatures. For a more microscopic "worm-like chain" model with bending rigidity $\kappa$ (assuming a small Debye screening length), we have $[27,28]$

$$
\ell \approx \frac{2 \kappa}{T}
$$

Here, and in what follows, we use boldface vectors $\mathbf{r}(s)$ to denote entire polymer configurations, and conventional vectors such as $\vec{r}(N d)$ to denote the position of a polymer endpoint.

To describe a DNA duplex, we bring the two strands together, neglect torsional rigidity and the helical nature of the bonding [28], and write the total free energy as

$$
\begin{array}{r}
F\left[\mathbf{r}_{1}(s), \mathbf{r}_{2}(s)\right]=\frac{K}{2} \int_{0}^{N d}\left[\left(\frac{d \mathbf{r}_{1}(s)}{d s}\right)^{2}+\left(\frac{d \mathbf{r}_{2}(s)}{d s}\right)^{2}\right] d s \\
+\int_{0}^{N d} U\left[\mathbf{r}_{1}(s)-\mathbf{r}_{2}(s)\right] d s
\end{array}
$$

where $U\left[\mathbf{r}_{1}(s)-\mathbf{r}_{2}(s)\right]$ is a potential which binds the nucleotides in different strands together. We assume that the complimentarity of the nucleotide sequences is sufficient to bring the strands into registry, but will otherwise neglect the effect of sequence disorder in the remainder of this section. If we now pass to sum and difference variables.

$$
\begin{gathered}
\mathbf{R}(s)=\frac{1}{2}\left[\mathbf{r}_{1}(s)+\mathbf{r}_{2}(s)\right] \\
\mathbf{r}(s)=\mathbf{r}_{2}(s)-\mathbf{r}_{1}(s)
\end{gathered}
$$

the coarse-grained free energy decouples, 


$$
\begin{gathered}
F[\mathbf{R}(s), \mathbf{r}(s)]=K \int_{0}^{N d} d s\left(\frac{d \mathbf{R}}{d s}\right)^{2}+\frac{1}{2} g \int_{0}^{N d} d s\left(\frac{d \mathbf{r}}{d s}\right)^{2} \\
+\int_{0}^{N d} d s U[\mathbf{r}(s)]
\end{gathered}
$$

where $g=K / 2$.

The partition function associated with (8) is a path integral of $e^{-F(\mathbf{R}, \mathbf{r}) / T}$ over the functions $\mathbf{R}(s)$ and $\mathbf{r}(s)$. The part associated with $\mathbf{R}(s)$ is simply the partition sum of an unconstrained Gaussian coil. The remaining functional integral over $\mathbf{r}(s)$ contains a denaturation transition. To pursue this point, we assume for $\operatorname{simplicity~that~} \mathbf{r}(s=0)=\overrightarrow{0}$ and $\mathbf{r}(s=N d)=\vec{r}$ (closed boundary conditions at one end, open at the other), and evaluate

$$
Z(\overrightarrow{0}, \vec{r} ; N)=\int_{\mathbf{r}(0)=\overrightarrow{0}}^{\mathbf{r}(N d)=\vec{r}} \mathcal{D} r \exp \left[-\frac{g}{2 T} \int_{0}^{N d}\left(\frac{d \mathbf{r}}{d s}\right)^{2} d s-\frac{1}{T} \int_{0}^{N d} U[\mathbf{r}(s)]\right] d s .
$$

As in polymer adsorption problems, it is helpful to view Eq. (9) as a Feynman path integral for a quantum mechanical particle in imaginary time [27]. Indeed, this partition function can be rewritten as a quantum mechanical matrix element [29],

$$
Z(\overrightarrow{0}, \vec{r} ; N)=\left\langle\vec{r}\left|e^{-\hat{H} N d / T}\right| \overrightarrow{0}\right\rangle,
$$

where the effective quantum Hamiltonian is

$$
\hat{H}=\frac{-T^{2}}{2 g} \nabla^{2}+U(\vec{r})
$$

and $|0\rangle$ and $\langle\vec{r}|$ are respectively ket and bra vectors localized at $\overrightarrow{0}$ and $\vec{r}$. Note that temperature plays the role of $\hbar$ and $g=\frac{1}{2} K=\frac{3 T}{2 \ell}$ represents the mass of a fictitious quantum mechanical particle in a potential $U(\vec{r})$. In the language of statistical mechanics, we have reduced this one-dimensional problem to diagonalizing a transfer matrix given by $\hat{T}=e^{-\hat{H} d / T}$. We shall focus on the particularly simple binding potential illustrated in Fig. 4, namely

$$
U(\vec{r})=\left\{\begin{array}{c}
\infty, r<c \\
-U_{0}, c<r<b \\
0, b<r,
\end{array}\right.
$$


with $U_{0}=V_{0} / d$, where $V_{0}$ is the average bonding energy per nucleotide. Upon inserting a complete set of energy eigenfunctions $|n\rangle=\phi_{n}(\vec{r})$ with eigenvalues $\epsilon_{n}$ into Eq. (10), we see that this conditional partition function may be rewritten as

$$
Z(\overrightarrow{0}, \vec{r} ; N)=\sum_{n}\left\langle\vec{r}\left|e^{-\hat{H} N d / T}\right| n\right\rangle\langle n \mid 0\rangle=\sum_{n} \phi_{n}^{*}(\overrightarrow{0}) \phi_{n}(\vec{r}) e^{-\epsilon_{n} N d / T}
$$

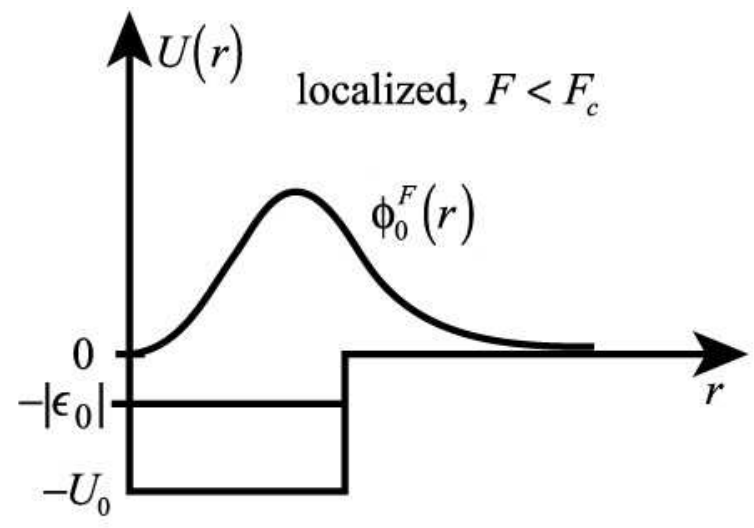

(a)

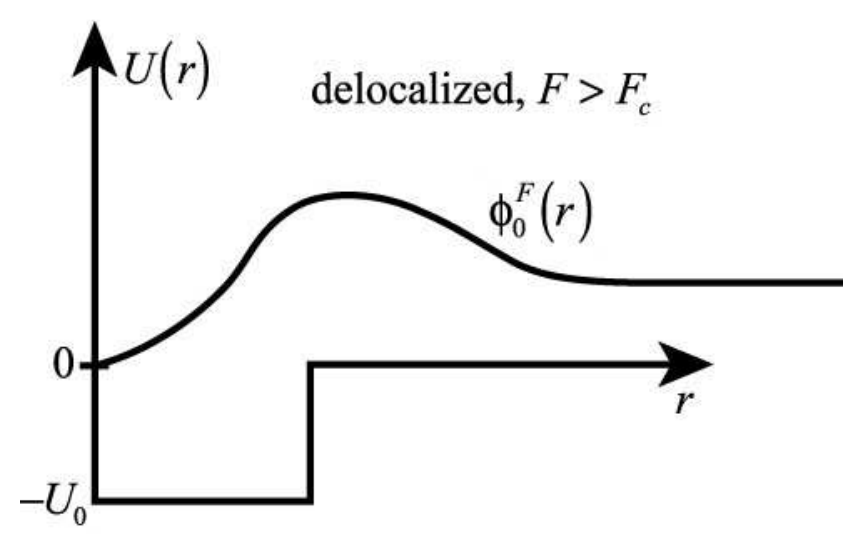

(b)

FIG. 4. Potential well and ground state eigenfunctions for forced-induced denaturation when (a) $F<F_{c}$ and (b) $F>F_{c}$. These (right) eigenfunctions give the probability of a separation $\vec{r}$ for the unzipped ends of the DNA. For $F<F_{c}$, the ground state eigenfunction decays exponentially to zero outside the well, which models the hydrogen bonds and stacking energies which hold base pairs together. For $F>F_{c}$, the eigenfunction tends to a nonzero constant for large $r$, and the two DNA strands fall apart. 
The ground state dominates in the limit $N \rightarrow \infty$, and we have

$$
Z(\overrightarrow{0}, \vec{r} ; N) \approx \phi_{0}(\overrightarrow{0}) \phi_{0}(\vec{r}) e^{-\epsilon_{0} N d / T}
$$

where the (nodeless) ground state eigenfunction can be chosen to be real.

The quantum mechanics of simple square well potentials like Eq. (12) in three dimensions is well understood [30]. We focus for simplicity on the case $c<<b$ (small hard core diameter) but the same qualitative discussion applies more generally. The key parameter controlling the eigenfunctions and eigenvalues is the dimensionless ratio

$$
Q=\frac{2 g U_{0} b^{2}}{T^{2}}
$$

of the well depth $U_{0}$ to the "zero point energy" $\frac{T^{2}}{2 g b^{2}}$. This quantum zero point energy represents the lost entropy associated with a confined DNA duplex. From the perspective of quantum mechanics, the loop excitations shown in Fig. 3b represent transient excursions (in imaginary time) out of the well. When $Q>>1$ (low temperatures), the binding energy dominates the thermal fluctuations favoring denaturation and there are many bound (i.e., localized) eigenstates of $\hat{H}$ beneath a continuum of extended eigenstates. As $Q$ decreases, more and more states delocalize until only the single localized ground state shown in Fig. (4a) is present. Eventually, at temperatures high enough so that $Q \leq Q^{*}$, even the ground state delocalizes [31]. For the simple problem discussed here $Q^{*}=\frac{\pi^{2}}{4}$ (i.e., $T_{m}=8 U_{0} b^{2} / \pi^{2} g$ ) [30]. However, a similar $Q^{*}$ of order unity is expected for a broad class of similar potentials. Near the transition temperature $T_{m}$, the ground state energy approaches the continuum quadratically with temperature $[27,30]$

$$
\epsilon_{0}(T) \approx-\text { const. }\left|T_{m}-T\right|^{2}
$$

where the melting temperature $T_{m}$ is defined by $Q\left(T_{m}\right)=Q^{*}$. For large $r$, the ground state wave function decays exponentially, $\phi_{0}(\vec{r}) \sim e^{-\kappa_{0}|\vec{r}|}$, where

$$
\kappa_{0}^{-1}(T)=\sqrt{\frac{-T^{2}}{2 g \epsilon_{0}(T)}} \sim \frac{1}{\left(T_{m}-T\right)}
$$


gives the spatial extent (in three dimensions) of the bubbles shown schematically in Fig. 3b. The number of base pairs $N_{b}$ in a bubble (see Fig. 3b) can be estimated by identifying $N_{b}$ with the value of $N$ needed to achieve ground state dominance in Eq. (12): We expect that boundary effects become unimportant when the number of base pairs exceeds the number in a typical bubble. The ground state will dominate once the number of nucleotides is larger than several bubble sizes. Since the energy of the first excited state for $Q \gtrsim Q^{*}$ is zero, we have

$$
Z(\overrightarrow{0}, \vec{r} ; N) \approx \phi_{0}(\overrightarrow{0}) \phi_{0}(\vec{r}) e^{\frac{\left|\epsilon_{0}\right| N d}{T}}\left[1+\mathcal{O}\left(e^{\frac{-\left|\epsilon_{0}\right| N d}{T}}\right)\right]
$$

from which it follows that

$$
N_{b} \approx \frac{T}{d\left|\epsilon_{0}(T)\right|} \sim \frac{1}{\left(T_{m}-T\right)^{2}}
$$

We should stress that the above results are obtained in an approximation which neglects self-avoidance effects important for large molecules close to $T_{m}$. We expect, however, qualitatively similar behavior even when self-avoidance is taken into account. We now use similar methods to treat force-induced denaturation in Section 3.

\section{FORCE-INDUCED DENATURATION}

A force $\vec{F}$ applied across the two strand endpoints (see Fig. 1) adds an energy $-\vec{F} \cdot \vec{r}$, and Eq. (9) becomes

$$
\begin{aligned}
Z(\overrightarrow{0}, \vec{r} ; N)=\int_{\mathbf{r}(0)=\overrightarrow{0}}^{\mathbf{r}(N d)=\vec{r}} D \mathbf{r}(s) \exp [ & -\frac{g}{2 T} \int_{0}^{N d}\left(\frac{d \mathbf{r}}{d s}\right)^{2} d s-\frac{1}{T} \int_{0}^{N d} U_{s}[\mathbf{r}(s)] d s \\
& \left.+\frac{\vec{F}}{T} \cdot \int_{0}^{N d} \frac{d \mathbf{r}}{d s} d s\right]
\end{aligned}
$$

We assume the attached strands of DNA can swivel freely to relax twist on an experimental time scale, so that we can neglect the helical nature of the duplex state when the force is applied. In addition to the force term (note that the lower limit does not contribute with our boundary conditions), we have added a subscript $s$ to the binding potential, $U[\mathbf{r}(s)] \rightarrow$ 
$U_{s}[\mathbf{r}(s)]$, to emphasize that the depth and size of potentials like that in Fig. 4 will depend on the particular base pair for heterogeneous DNA. After taking over results for a virtually identical problem [32] of flux line depinning by a columnar pin in a transverse magnetic field in high $T_{c}$ superconductors, we obtain $[9,10]$

$$
T \frac{\partial Z(\overrightarrow{0}, \vec{r} ; N)}{\partial N d}=\frac{-T^{2}}{2 g}\left(\vec{\nabla}-\frac{\vec{F}}{T}\right)^{2} Z(\overrightarrow{0}, \vec{r} ; N)+U_{N d}(\vec{r}) Z(\overrightarrow{0}, \vec{r} ; N),
$$

which can be integrated (subject to the boundary condition $Z(\overrightarrow{0}, \vec{r} ; 0)=1$ ) to give a result with the form of Eq. (10), where

$$
\hat{H}(\vec{F})=\frac{-T^{2}}{2 g}\left(\vec{\nabla}-\frac{\vec{F}}{T}\right)^{2}+U_{N d}(\vec{r}) .
$$

Equation (20) with an $s$-dependent potential $U_{s}[\vec{r}]$ can be studied (via a mapping onto a Burgers equation) by the methods of Ref. [33], where it arose in a study of vortex depinning from fragmented columnar pins. In this section, we neglect the $s$-dependence (letting $\left.U_{N d}(\vec{r}) \rightarrow U(\vec{r})\right)$ and illustrate force-induced denaturation for the homopolymer duplex discussed in Sec. 2. Because $\vec{F}$ appears as a constant imaginary "vector potential," Eq. (21) represents a non-Hermitian generalization of Schroedinger's equation. To establish the existence of a first-order transition, we write the solutions of (20) using the right and left eigenfunctions $\left\{\phi_{n R}^{F}(\vec{r})\right\}$ and $\left\{\phi_{n L}^{F}(\vec{r})\right\}$ (with a set of common eigenvalues $\left.\left\{E_{n}(F)\right\}\right)$ of $\hat{H}(\vec{F})$,

$$
Z(\overrightarrow{0}, \vec{r} ; N)=\sum_{n} \phi_{n L}^{F}(\overrightarrow{0}) \phi_{n R}^{F}(\vec{r}) e^{-E_{n}(F) N d / T} .
$$

We again invoke ground-state dominance and focus on the behavior of the lowest eigenvalue, which determines the duplex free energy per unit length $a(F)$ via

$$
a(F)=-T \lim _{N \rightarrow \infty} \frac{1}{N d} \ln [Z(N, F)],
$$

where $Z(N, F)$ is defined by integrating over $\vec{r}$,

$$
Z(N, F)=\int d^{3} r Z(\overrightarrow{0}, \vec{r} ; N),
$$

since we work at constant force instead of constant extension. 
For $F$ less than a critical value $F_{c}$ (determined below), we can obtain the (right) eigenfunctions $\phi_{n R} \vec{F}_{r}(\vec{r})$ for $\vec{F} \neq 0$ from localized eigenfunctions $\phi_{n}(\vec{r})$ for the Hermitian case $\vec{F}=0$ via a "gauge transformation" [32]. Indeed, it is easy to check that

$$
\phi_{n R}^{\vec{F}}(\vec{r})=e^{\frac{\vec{F} \cdot \vec{r}}{T}} \phi_{n}(\vec{r})
$$

solves

$$
\hat{H}(\vec{F}) \phi_{n R}^{\vec{F}}(\vec{r})=E_{n} \phi_{n}^{F}(\vec{r})
$$

(provided $\phi_{n}(\vec{r})$ satisfies $\left.\hat{H}(\overrightarrow{0}) \phi_{n}(\vec{r})=\epsilon_{n} \phi_{n}(\vec{r})\right)$ with the same eigenvalue, $E_{n}=\epsilon_{n}$. In particular, we obtain from Eq. (23) a negative $\vec{F}$-independent free energy per unit length $a=\epsilon_{0}=-\left|\epsilon_{0}\right|$ for small $\vec{F}$. If, however, a localized ground-state eigenfunction for the Hermitian $\vec{F}=0$ problem decays like $\phi_{0}(\vec{r}) \sim e^{-\kappa_{0}|\vec{r}|}$ for large $r$, the new eigenfunctions (25) are only normalizable provided $F \leq F_{c}(T)=T \kappa_{0}(T)$. Note that $\phi_{0 R}^{\vec{F}}(\vec{r})$, which gives the probability of finding an extension $\vec{r}$, [32] is displaced in the direction of $\vec{F}$, as indicated schematically in Fig. 4a. To determine what happens for $F>F_{c}$, we check for a nodeless extended ground-state wavefunction, as indicated in Fig. 4b. If $\phi_{0 R}^{F}(\vec{r}) \rightarrow$ const. $>0$ for large $r$ (i.e., the DNA duplex falls apart), and $\lim _{r \rightarrow \infty} U(\vec{r})=0$, evaluating Eq. (22) in this limit gives $\hat{H}(F) \phi_{O R}^{F}(\vec{r})=\frac{-F^{2}}{2 g} \phi_{O R}^{F}(\vec{r})$, so that $a(F)=E_{0}(F)=\frac{-F^{2}}{2 g}$. The two free energies $a(F)$ for $F<F_{c}$ and $F>F_{c}$ are plotted in Fig. 5 [32]. At $F_{c}$ the two curves intersect at a nonzero angle, the classic signature of a first-order phase transition. The latent heat of this transition might be observable in a solution of many DNA duplexes pulled apart by, say, beads of opposite charge in an electric field. Since the first-order transition curve $F_{c}(T)$ is given by $-F_{c}^{2} / 2 g=-\left|\epsilon_{0}(T)\right|$, where $\epsilon_{0}$ is the smallest eigenvalue of the transfer matrix for $F=0$, we find using Eq. (15) that

$$
F_{c}(T) \propto \text { const. }\left|T_{m}-T\right|
$$

near the melting transition for this model. 


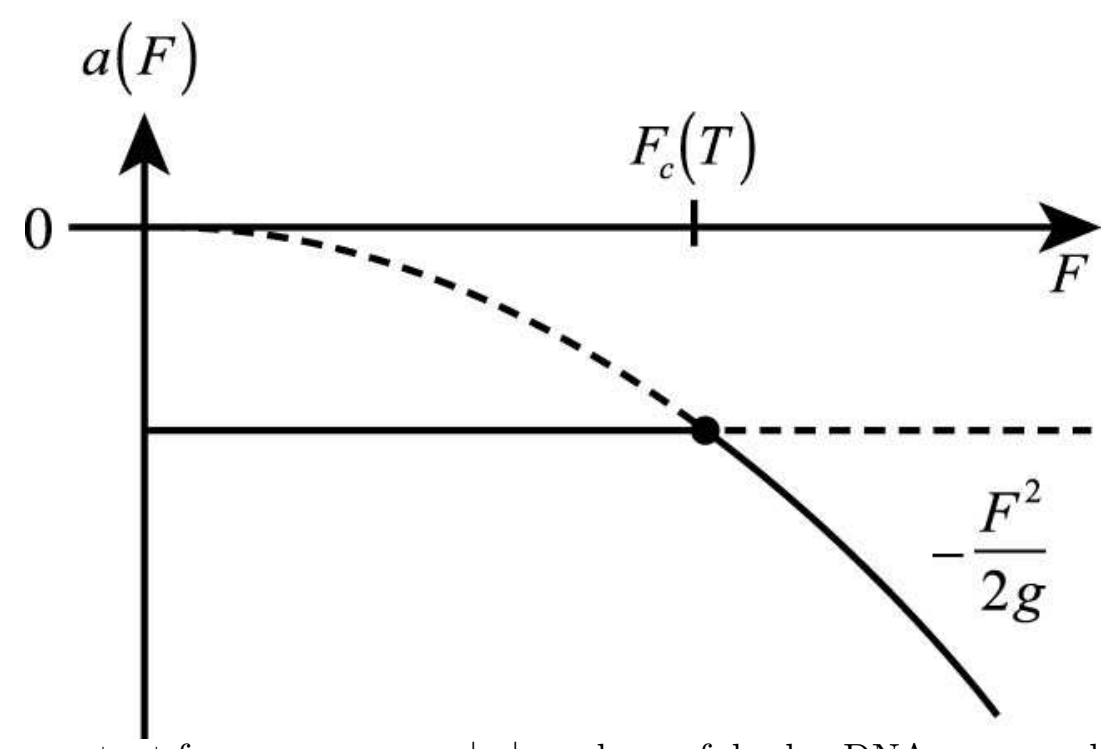

FIG. 5. The constant free energy $a=-\left|\epsilon_{0}\right|$ per base of duplex DNA compared to the parabolic free energy $a(F)=-F^{2} / 2 g$ appropriate for two separated strands. See the text for a discussion of more sophisticated freely-jointed chain and worm-like chain models of this free energy. The two free energies cross with discontinuous slope at $F_{c}(T)$, the location of the first-order unzipping transition.

Even though force-induced denaturation is a first-order transition, there are diverging precursors in the form of DNA unzipping (see Fig. 1). The probability of an endpoint separation $|\vec{r}|$ in the limit $N \rightarrow \infty$ is given integrating $|\vec{r}|$ weighted by the ground state eigenfunction,

$$
\begin{aligned}
\phi_{0 R}^{\vec{F}} & =e^{\frac{\vec{F} \cdot \vec{r}}{T}} \phi_{0}(\vec{r}) \\
& \simeq e^{\frac{\vec{F} \cdot \vec{r}}{T}} e^{-\kappa_{0}|r|} .
\end{aligned}
$$

It is then straightforward to show that $\langle|\vec{r}|\rangle$ diverges as $F \rightarrow F_{c}^{-}(T)[32]$

$$
\langle|\vec{r}|\rangle \sim \frac{1}{F_{c}(T)-F}
$$

Upon examining corrections to ground state dominance as in the analysis of Eq. (19), we find that the average number $m$ of unzipped monomers diverges, as shown in Fig. 6a,

$$
\begin{aligned}
\langle m\rangle \simeq & \frac{T}{d\left(\left|\epsilon_{0}\right|-F^{2} / 2 g\right)} \\
& \sim \frac{1}{F_{c}(T)-F} .
\end{aligned}
$$



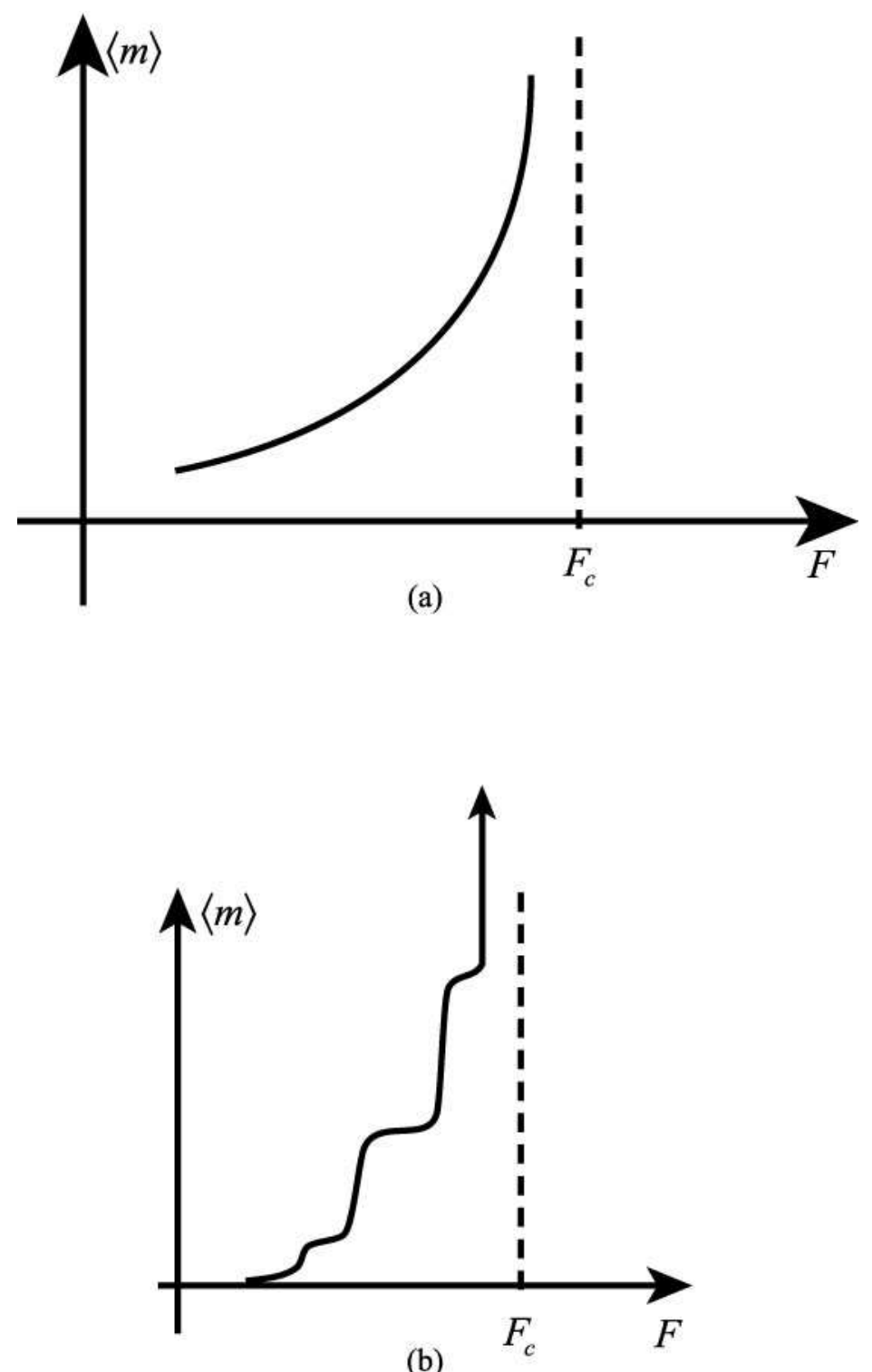

FIG. 6. Thermal average $\langle m\rangle$ of the location of the unzipping fork. (a) Divergence of $\langle m\rangle$ at $F_{c}$ for a homopolymer. (b) Jumps and plateaus in $\langle m\rangle$ (with, on average, a much strong divergence) in the presence of sequence disorder.

The first-order force-induced DNA denaturation transition can be understood more generally, independent of the mapping onto quantum mechanics. If $a_{1}(T, F)$ is the free energy per unit length of one of the single-stranded DNA "handles" shown in Fig. 7, and $a_{0}(T)$ is the (force-independent) free energy per unit length of the double-stranded DNA which coexists in a macroscopically unzipped state, then the condition for two-phase coexistence across the "meniscus" or unzipping fork at the critical force $F_{c}$ is [14] 


$$
a_{0}(T)=2 a_{1}\left(F_{c}, T\right) .
$$

The physics of the handle free energy $a_{1}(F, T)$ is relatively simple, provided the force is large enough to neglect self-hybridization and self-avoidance of the single-stranded DNA. For example, we could take $a_{1}(T, F)$ to be the free energy of a freely-jointed chain with persistance length $\ell[25]$

$$
a_{1}(T, F) \propto-\frac{T}{\ell} \ln \left(\frac{T \sinh (F \ell / T)}{F \ell}\right),
$$

which is proportional to $-\ell F^{2} / T$ for small $F$. Alternatively one could use a more sophisticated "worm-line chain" approximation valid over a larger range of forces [34]. With an acceptable approximation to $a_{1}(T, F)$ in hand (if necessary, this function could be obtained directly from integrating single strand force-extension curves [35]), one can use the experimentally determined phase boundary $F_{c}(T)$ for unzipping in conjunction with Eq. (31) to explore the temperature dependence of the free energy $a_{0}(T)$ of double-stranded DNA.

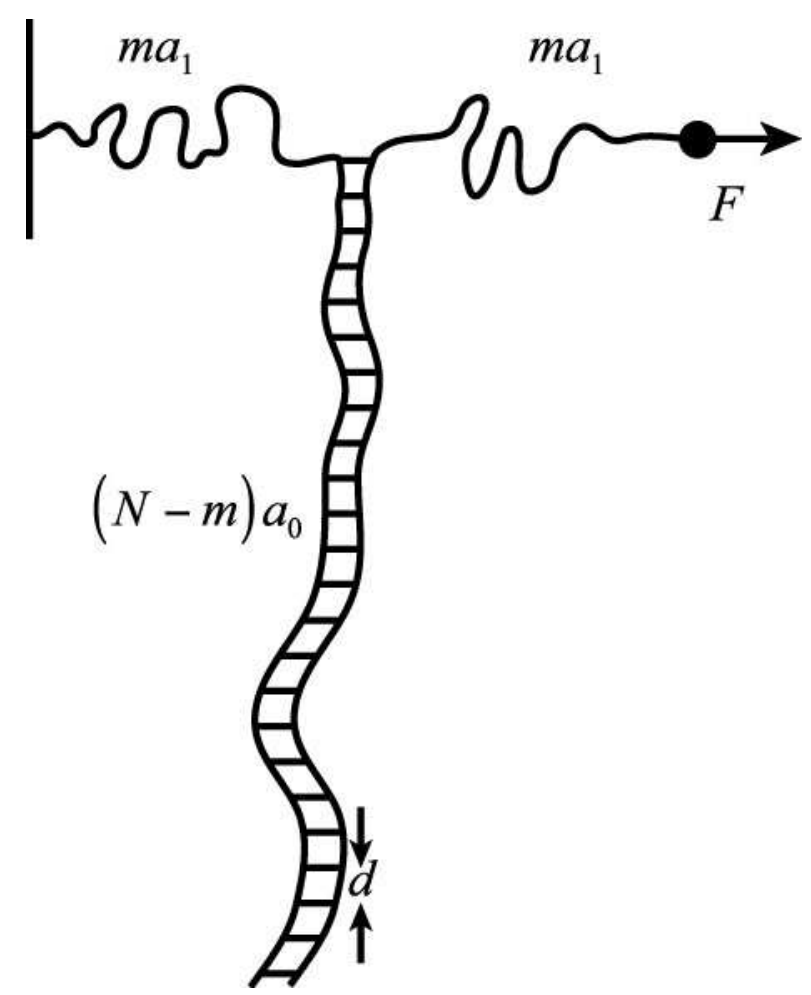

FIG. 7. Two-phase coexistence of duplex DNA with free energy oer base pair $a_{0}$ and two unzipped "handles," each with free energy per base $a_{1}$. 


\section{UNZIPPING WITH SEQUENCE HETEROGENEITY}

The effect of sequence heterogeneity on thermal denaturation of DNA at zero force is a subtle and still not completely resolved problem, [19,23,24]. Understanding inhomogeneous base pairing energies near the first-order force-induced unzipping transition appears to be more tractable. The integrated effect of sequence randomness on the energy landscape for long strands of DNA does not change the underlying first-order transition, because it only

produces corrections of order $\frac{1}{\sqrt{N}}$ to the free energies per unit length discussed above, where $N$ is the total number of base pairs. However, sequence randomness has drastic consequences for quantities like $\langle m\rangle$ which characterize the unzipping process itself $[9,14]$. Here, we simply sketch the overall picture. Detailed calculations and justifications for approximations (such as the neglect of bubbles in DNA) can be found in Ref. [14].

Consider the energy $\epsilon(m)$ associated with the conditional partition sum of a DNA duplex which has been unzipped by $m$ base pairs. If the DNA is a homopolymer, consisting exclusively of A:T or G:C base pairs, the total energy can be read off from Fig. 7,

$$
\begin{aligned}
\mathcal{E}(m) & =2 m a_{1}+(N-m) a_{0} \\
& =a_{0} N+f m
\end{aligned}
$$

where

$$
f \equiv 2 a_{1}(F)-a_{0}
$$

can be expanded to give $\left.f \approx c \frac{2 d a_{1}}{d F}\right|_{F_{c}}\left(F_{c}-F\right)$ near the unzipping transition. If we now add sequence randomness, a reasonable model for the energy of unzipping is

$$
\begin{aligned}
\Delta \mathcal{E}(m) & \equiv \mathcal{E}(m)-N a_{0} \\
& =f m+\int_{0}^{m} d m^{\prime} \eta\left(m^{\prime}\right),
\end{aligned}
$$

where deviations from the average A:T or G:C content are represented by the function $\eta\left(m^{\prime}\right)$ in a convenient continuum notation. To the extent that most DNA sequences which code 
for proteins have deviations from the average $\mathrm{G}: \mathrm{C} / \mathrm{A}: \mathrm{T}$ content describable as a random walk [36], we expect that the statistical properties of $\eta(m)$ at large distances are those of a white-noise random variable,

$$
\overline{\eta(m) \eta\left(m^{\prime}\right)}=\Delta_{0} \delta\left(m-m^{\prime}\right)
$$

where the overbar represents a "disorder average" over all possible sequences of base pairs. Because G:C and A:T pairing energies differ by an amount of order $T, \Delta_{0} \simeq T^{2}$, where we have used the temperature $T \simeq 293-310^{\circ} \mathrm{K}$ characteristic of most experiments to characterize this quantity. The partition function associated with this simple one-dimensional model involves integrating over all possible unzipping lengths $m$,

$$
\mathcal{Z}(f)=\int_{0}^{\infty} d m e^{-\Delta \mathcal{E}(m) / T}
$$

The unzipping energy in Eq. (35) is plotted for a particular base pair sequence (with $\Delta_{0}=T^{2}$ ) in Fig. 8, with $f / T=0.01$. The straight line is the energy of a homopolymer duplex with $\Delta_{0}=0$ and

$$
\Delta \mathcal{E}(m)=f m
$$

In this case we find immediately from Eq. (37) that the thermally averaged degree of unzipping is

$$
\begin{aligned}
\langle m\rangle & =-T \frac{d \ln \mathcal{Z}(f)}{d f} \\
& =\frac{T}{f} \sim \frac{1}{\left(F_{c}-F\right)},
\end{aligned}
$$

in agreement with Eq. (30). There are large fluctuations about this average value, as one can check by evaluating

$$
\sqrt{\left\langle(m-\langle m\rangle)^{2}\right\rangle}=T / f=\langle m\rangle .
$$




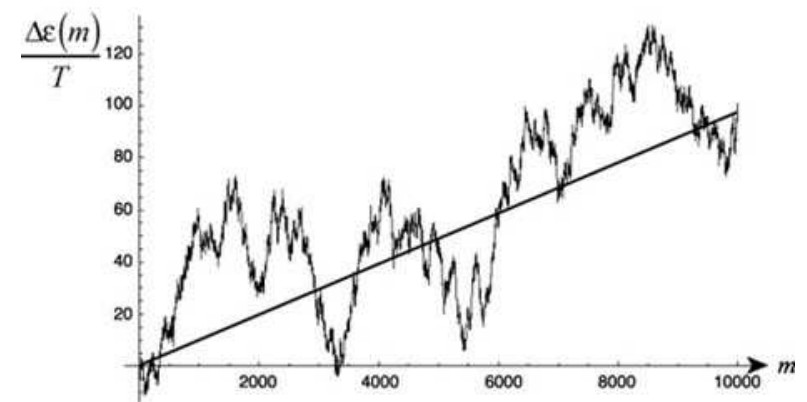

FIG. 8. Energy landscape for $F \leq F_{c}$ for a homopolymer duplex (straight line) and with the addition of inhomogeneous base pairing energies due to a particular DNA sequence (jagged line). Here we take $\Delta_{0}=T^{2}$ and $f / t=0.01$.

The rugged landscape in Fig. 8 for $\Delta_{0} \neq 0$ is more interesting. Although the positive background slope determined by $f$ insures that the DNA remains macroscopically unzipped, the integrated effect of sequence randomness produces deviations from a straight line which scale like $\sqrt{m}$. These fluctuations lead to deep minima at nonzero $m$ with large energy barriers in between. A downward fluctuation in this "integrated random walk" energy landscape at position $m$ corresponds to an energy of order

$$
\Delta \epsilon(m) \simeq f m-\sqrt{\Delta_{0} m}
$$

Upon minimizing over $m$, we obtain the estimate

$$
\overline{\langle m\rangle} \sim \frac{\Delta_{0}}{f^{2}} \sim \frac{1}{\left(F_{c}-F\right)^{2}}
$$

a result confirmed by more elaborate calculations $[9,14]$. Here, the overbar represents an average over a quenched random distribution of DNA sequences. It can also be shown that thermal fluctuations about this average are more constrained than for homopolymers, in the sense that

$$
\sqrt{\overline{\left\langle(m-\langle m\rangle)^{2}\right\rangle}} \sim \frac{1}{f^{3 / 2}} \sim \overline{\langle m\rangle}^{3 / 4}
$$

Thus, in contrast to homopolymers, where $\left\langle(m-\langle m\rangle)^{2}\right\rangle /\langle m\rangle^{2}=\mathcal{O}(1)$ we now have 


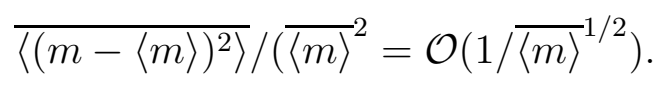

A typical energy barrier associated with disorder-dominated unzipping is $f\langle m\rangle+\sqrt{\Delta_{0}\langle m\rangle} \sim$ $\frac{\Delta_{0}}{f}$. Results such as (42) apply when this scale exceeds $T$, i.e., for $f$ less than a crossover force $f_{x}$,

$$
f<f_{x}=\frac{\Delta_{0}}{T}
$$

Because $\Delta_{0}=\mathcal{O}\left(T^{2}\right)$ is large for $\mathrm{G}: \mathrm{C}$ and $\mathrm{A}: \mathrm{T}$ base pairs, the range of forces where sequence heterogeneity dominates the physics is the large region bounded by the left-hand dashed line in Fig. 2. Note that disordered dominated prediction for $\overline{\langle m\rangle}$ in Eq. (42) diverges with a power which is twice as large as the result (39) appropriate for thermally equilibrated homopolymers.

Results such as Eq. (42) apply only to a quenched average over an entire library of different DNA sequences unzipped in parallel. We can understand the behavior for $F \lesssim$ $F_{c}(T)$ for the more experimentally accessible case of a particular squence from Fig. 9, which shows the energy landscape for two identical sequences with the biases, $f / T=0.01$ and $f / T=0=0.006$. The degree of unzipping $\langle m\rangle$ is dominated by the minimum indicated by an arrow in each case. Somewhere between $f=0.01$ and $f=0.006$, the average of $\langle m\rangle$ jumps from one minimum to the next. More detailed calculations [14] reveal an entire sequence of jumps and plateaus, as illustrated in Fig. 6b. Although a best fit to a power law of this irregular curve should reveal the exponent of Eq. (42), the plateaus and jumps themselves represent a rough "fingerprint" of the individual sequence. See Ref. [14], where the statistical distribution of plateaus and jumps are evaluated using the techniques of LeDoussal et al. [37]. 


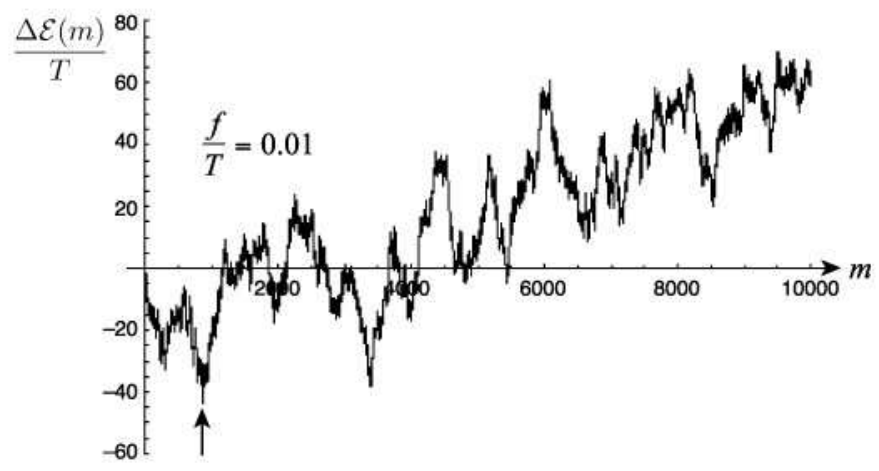

(a)

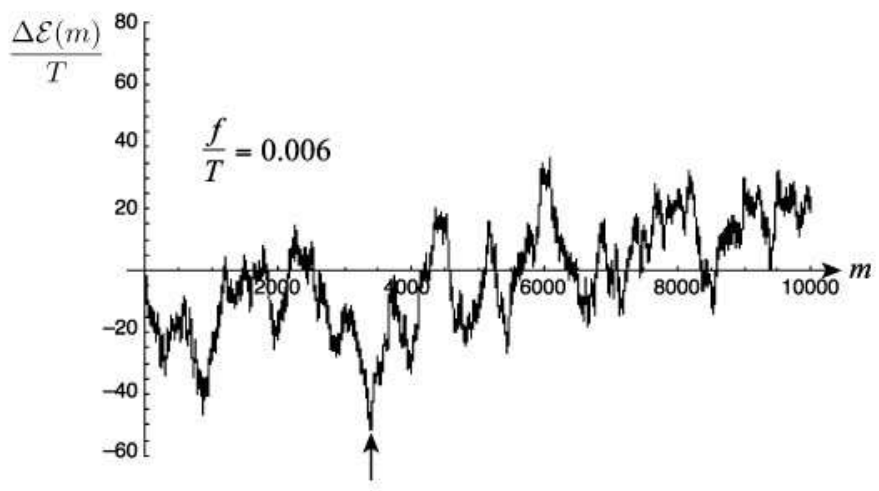

(b)

FIG. 9. Energy landscapes two identical DNA sequences with $\Delta_{0}=T^{2}$ and (a) $f / T=0.01$ and (b) $f / T=0.006$. The arrow indicates the energy minimum which dominates the value of $\langle m\rangle$ in each case.

\section{DYNAMICS OF UNZIPPING}

What happens for $F \gtrsim F_{c}(T)$ ? In Figure 10 we show an energy landscape (for the same sequence as in Fig. 8) with $f / T=-0.01$, together with the purely downhill landscape of the corresponding homopolymer. Because the average free energy per base pair of the DNA duplex exceeds the combined free energies per base of the two single-stranded DNA "handles" in Fig. 7, the system is unstable to complete unzipping. It is then appropriate to discuss the dynamical process by which unzipping proceeds on this downhill path. 


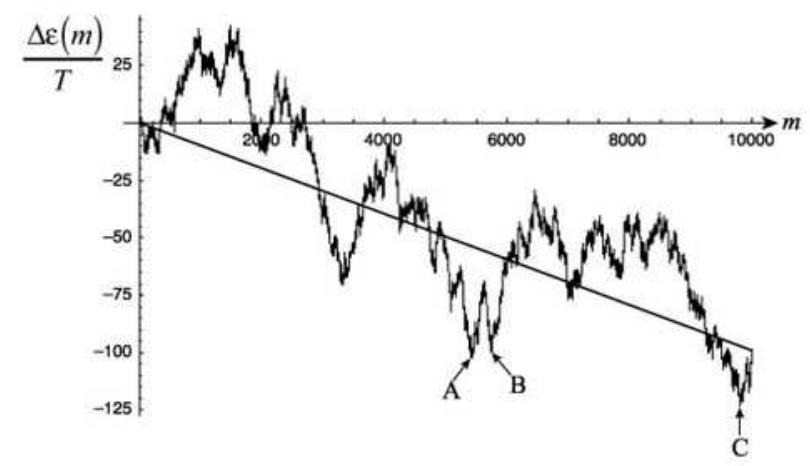

FIG. 10. Downhill energy landscape appropriate for are $F \geq F_{c}(T)$ Although the overall slope is negative, there are many traps, such as those at positions A, B, and C. Energy barriers between these traps scale as $\sqrt{\Delta_{0} m}$, where $m$ is the number of bases between traps.

Subject to a number of simplifying conditions discussed in Ref. [14], the time dependence of the unzipping fork can be described by an overdamped Langevin equation,

$$
\frac{d m(t)}{d t}=-\Gamma \frac{d \mathcal{E}(m)}{d m}+\zeta(t)
$$

where $\Gamma$ is a damping constant $(\Gamma=1 /(\tau T)$, where $\tau$ is a microscopic relaxation time) and the noise correlations are

$$
\left\langle\zeta(t) \zeta\left(t^{\prime}\right)\right\rangle=2 T \Gamma \delta\left(t-t^{\prime}\right)
$$

Upon substituting for the energy landscape $\mathcal{E}(m)$ from Eq. (35), we consider the case $f<0$ and obtain

$$
\frac{d m(t)}{d t}=\Gamma|f|-\Gamma \eta(m)+\zeta(t)
$$

which is the equation of motion for a particle with coordinate $m(t)$ executing one-dimensional biased diffusion in a random force field proportional to $\eta(m)$.

A great deal is known about this problem [38-40]. In the absence of sequence heterogeneity $\left(\Delta_{0}=0\right.$ in Eq. $\left.(36)\right), m(t)$ exhibits diffusion with drift at long times, i.e.,

$$
\begin{gathered}
\lim _{t \rightarrow \infty} \frac{\langle m(t)\rangle}{t}=v \\
\lim _{t \rightarrow \infty} \frac{\left\langle[m(t)-\langle m(0)\rangle]^{2}\right\rangle}{t}=2 D
\end{gathered}
$$


with a well-defined drift velocity $v=\Gamma|f|$ and diffusion constant $D=\Gamma T$. This is the expected behavior for the straight line homopolymer energy landscape shown in Fig. 10. Sequence heterogeneity, however, has a dramatic effect on the dynamics unless $|f|$ is large. Indeed, the integrated random walk landscape in Fig. 10 has many deep minima, even though the average slope is negative. When $|f|$ is small, typical energy barriers to travel a distance $m$ scale like $\sqrt{\Delta_{0} m}$, as is reflected in the small barrier connecting the minima labelled A and B and the much larger barrier between these minima and the more distant minimum C. A detailed analysis [38-40] reveals three types of anomalous dynamics, depending on the dimensionless parameter

$$
\mu=\frac{2 T|f|}{\Delta_{0}}
$$
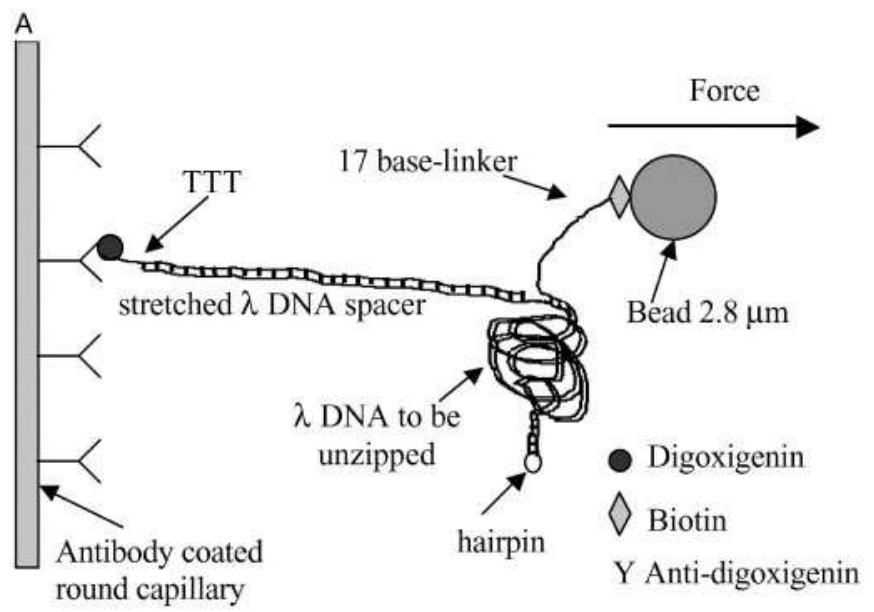

FIG. 11. Experimental setup for the force DNA unzipping experiments of Danilowicz et al. [42]. The unzipping trajectories of dozens of genetically identical lambda phage DNAs attached to a thin capillary tube are tracked. A constant force is generated by attaching magnetic beads and placing the apparatus in a magnetic field gradient.

Right at the unzipping transition, $\mu=|f|=0$ and the unzipping fork wanders subdiffusively according to [39]

$$
\overline{\left\langle|m(t)-m(0)|^{2}\right\rangle} \underset{t \rightarrow \infty}{\simeq} \frac{T^{4}}{\Delta_{0}^{2}} \ln ^{4}(t / \tau)
$$


because of trapping effects arising from the large energy barriers. The conventional diffusion constant $D$, as defined by Eq. (50), vanishes. When $0<\mu<1$, the unzipping fork drifts downhill, but does so sublinearly with time,

$$
\overline{\langle m(t)\rangle} \underset{t \rightarrow \infty}{\simeq} \text { const. } t^{\mu}
$$

so the usual drift velocity $v$ defined by (49) vanishes, with an additional anomaly in the spread about this average drift $[39,40]$. When this bias is large enough so that $1<\mu<2$, one recovers a well-defined drift velocity $v$, but with superdiffusive spreading,

$$
\overline{\left\langle[m(t)-\langle m(t)\rangle]^{2}\right\rangle} \underset{t \rightarrow \infty}{\sim} t^{2 / \mu} .
$$

Conventional diffusion with drift is only recovered for forces large enough so that $\mu>2$, i.e., for

$$
-f>f_{x}^{\prime}=\frac{\Delta_{0}}{T}
$$

This critical force is the same order of magnitude as the crossover scale defined below the unzipping transition by Eq. (45) and leads to the right-hand dashed line in Fig. 2. The parameters of DNA are such that anomalous unzipping appears in a very large range (compared to typical disorder effects in conventional critical phenoman [41]), roughly $\frac{1}{2} F_{c} \lesssim F \leq \frac{3}{2} F_{c}$. 


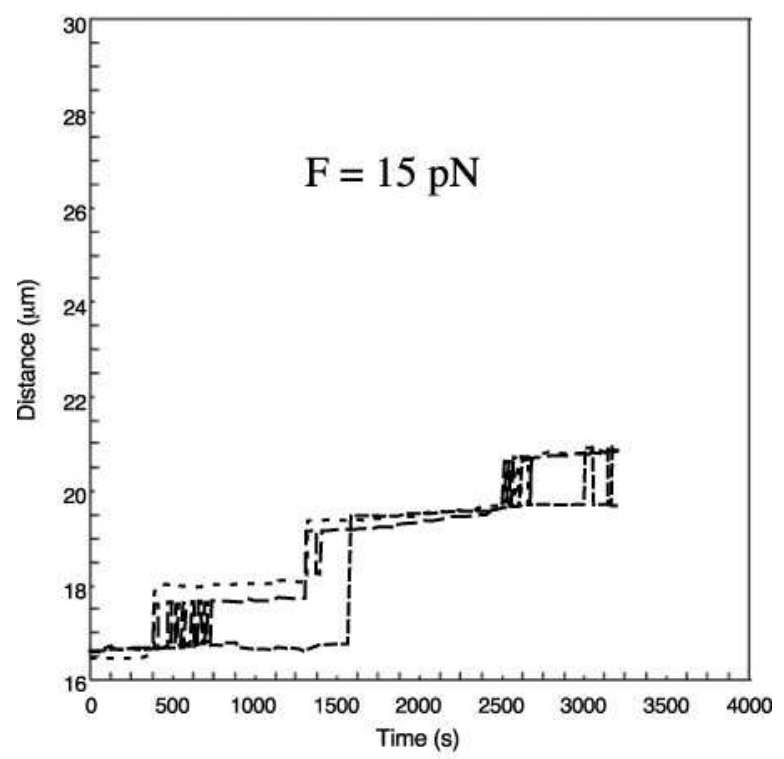

(a)

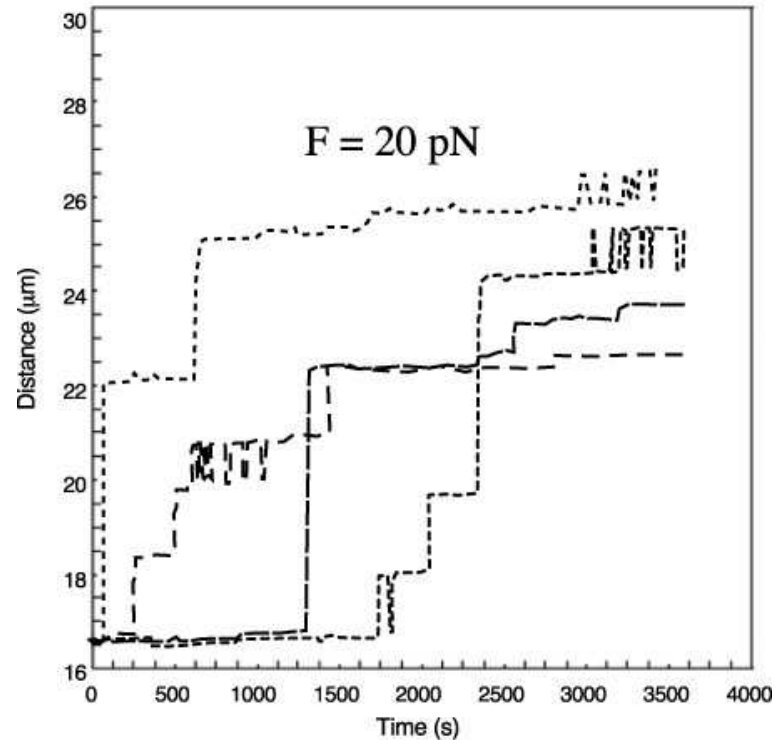

(b)

FIG. 12. Unzipping histories obtained from the apparatus of Fig. 11 for (a) three beads with $F=15 p N$ and (b) four beads with $F=20 p N$. Note the long-time scale (of order 1 hour) and the many jumps and plateaus. The oscillations arise when the unzipping fork encounters two nearly degenerate energy minima. The same plateaus recur for different beads at the same force, suggesting that these are due to sequence disorder (from Ref. [42]). 


\section{PAUSES, JUMPS AND CONCLUDING REMARKS}

Experiments which study the mechanical denaturation of lambda phage DNA at constant force have recently been carried out in the group of Mara Prentiss [42]. Digoxigenin linkers attach several dozen identical copies of this DNA along a long thin capillary tube. Each DNA to be unzipped is offset by an additional DNA spacer (see Fig. 11). Biotin links the other end of the DNA to magnetic beads. Unzipping at constant force is achieved by applying a uniform magnetic field gradient, causing the beads to stretch out at right angles to the capillary tube, like a series of flags on a flagpole.

Figure $12 \mathrm{a}$ records some unzipping histories at $T=20^{\circ} \mathrm{C}$ for three different beads at different heights on this "flagpole" with $F=15 p N$, corresponding approximately to the critical unzipping force for the front half of the sequence for phage lambda. (The back half of this DNA has a lower G:C content.) The beads are well separated, and are clearly subject to microscopically different thermal histories. Nevertheless, there are distinctive pauses (some lasting 15 minutes or more) and jumps common to the different beads. As the individual DNA's unzip, the beads occasionally jump back and forth, suggesting two nearly degenerate minima in the energy landscape about 1000 base pairs apart. This behavior is similar to that found for much shorter RNA hairpains in Ref. [5]. The long pauses before unzipping resumes are consistent with large energy barriers, of order 20-30 T. In contrast, unzipping at a slow constant velocity is an imperative in the work of Bockelmann et al. [6-8]. Because these experiments are effectively at constant extension, the force in this case fluctuates as needed about $F_{c}(T)$ to insure that all energy barriers are overcome.

Many of the pauses and local "two-level system" oscillations found by the Prentiss group coincide with those predicted for phage lambda DNA [42], using energy landscapes obtained from the base pairing and stacking energies of Santa Lucia et al. [43]. Overall, these experiments seem consistent with important aspects of the theory reviewed here, such as large energy barriers and sequence-specific pause points. We should stress that longer run times or higher temperatures are necessary to obtain true thermodynamic equilibrium. Figure 
$12 \mathrm{~b}$ shows the unzipping history of four different beads at $F \simeq 20 p N$. There are again jumps between common plateaus as well as oscillations suggesting nearly degenerate minima. Although $20 p N$ should be above the thermodynamic critical force $F_{c}(T)$, the DNA nevertheless fails to unzip completely on experimental time scales, presumably due to the ruggedness of "downhill" landscapes such as that in Fig. 10. Predictions such as Eq. (42) are only be applicable to systems in true thermodynamic equilibrium.

Experimental checks of phase diagrams such as that in Fig. 2 and the associated physical predictions would be of considerable interest. As mentioned in Sec. 3, the phase diagram itself can be used to directly measure the temperature-dependent free energy of the DNA duplex at zero force. Analysis of this free energy is simplified if a theory of the unzipped handles is available. Analytic theories require forces large enough to stretch out the handles and prevent hairpins created by self-hybridization. The self-hybridization of ssDNA (for $F \lesssim 10 \mathrm{pN}$ ) indicated in the experiments of Maier et al. [44] could be reduced by introducing single stranded binding proteins to smooth out the heterogeneity of the handles.

The assumption (36) of short-range correlations in the sequence is an approximation valid only for simple "coding DNA" in bacteria or viruses. The introns or so-called "junk DNA" present in many eukaryotic organisms may be better described by [36]

$$
\overline{\eta(m) \eta\left(m^{\prime}\right)} \sim \frac{1}{\left|m-m^{\prime}\right|^{2-2 \beta}},
$$

with $\beta>1 / 2$. Although we are unaware of detailed calculations, a straightforward generalization of the argument leading to Eq. (42) now gives [9]

$$
\overline{\langle m\rangle} \sim 1 / f^{\frac{1}{1-\beta}} .
$$

For $\beta \leq 1 / 2$, we expect that predictions for sequences with short-range correlations will apply. The exponent $\beta=0.55$ seems to describe the energy correlations of some intron-rich sequences [36].

Ideas similar to those discussed here can also be applied to force-induced denaturation $[45,14]$ of the multiple stem-loop structures which define the secondary structure of compli- 
cated RNA enzymes. Here, however, strings of RNA hairpins are unzipped simultaneously and the discrete jumps and plateaus in $\langle m\rangle$ near the unzipping force are averaged out.

\section{ACKNOWLEDGMENTS}

Virtually all the theoretical work described here is contained in the Ph.D. thesis of my former student, David Lubensky. I am fortunate to have such students. I am grateful as well to Claudia Danilowicz, Mara Prentiss and other experimenters for their determination to test some of the predictions sketched here. Finally, I would like to thank D. Branton, R. Bundschuh, T. Hwa, and Y. Kafri for helpful conversations on this material. This work was supported by the National Science Foundation, through grant DMR 0231631 and in part through the Harvard Materials Research Laboratory, via Grant DMR 0213805. 


\section{REFERENCES}

[1] For a brief review, see C. Bustamante, Z. Bryant and S.B. Smith, Nature 421, 423 (2003).

[2] F. Crick, Nature 227, 561 (1970).

[3] M. Rief, M. Gautel, F. Oesterhelt, J.M. Fernandez, and J. Gaub, Science 276, 1109 (1997).

[4] T.R. Strick, V. Croquette, and D. Bensimon, PNAS 95, 10,579 (1998).

[5] J. Liphardt, B. Onoa, S.B. Smith, I. Tinoco, Jr., and C. Bustamante, Science 292, 733 (2001).

[6] U. Bockelmann, Bessevaz, and F. Heslot, Phys. Rev. Lett. 79, 4489 (1997); Phys. Rev. E58, 2386 (1998).

[7] B. Essevaz-Roulet, U. Bockelmann, and F. Heslot, Proc. Natl. Acad. Sci. USA 94, 11935 (1997).

[8] U. Bockelmann, P. Thomen, B. Essevaz-Roulet, V. Viasnoff, and F. Heslot, Biophys. J. 82, 1537 (2002).

[9] D.K. Lubensky and D.R. Nelson, Phys. Rev. Lett. 85, 1572 (2000).

[10] S.M. Bhattacharjee, J. Phys. A48, L423 (2000).

[11] K.L. Sebastian, Phys. Rev. E62, 1128 (2000).

[12] S. Cocco, R. Monasson, and J.F. Marko, Proc. Natl. Acad. Sci. USA 98, 8608 (2001); Phys. Rev. E65, 0141907 (2002).

[13] D. Morenduzzo, S. Bhattacharjee, S. Maritan, E. Orlandini, and F. Seno, Phys. Rev. Lett. 88, 028102 (2002).

[14] D.K. Lubensky and D.R. Nelson, Phys. Rev. E65, 031917 (2002). 
[15] B. Alberts, D. Bray, J. Lewis, M. Ruff, K. Roberts, and J.D. Watson, Molecular Biology of the Cell (Garland, New York, 1994).

[16] P.G. deGennes, Rev. Mod. Phys. 57, 827 (1985).

[17] D. Poland and H.A. Scheraga, Theory of Helix-Coil Transitions in Biopolymers (Academic, New York, 1970).

[18] F.W. Wiegel, in Phase Transitions and Critical Phenomena, Vol. 7, edited by C. Domb and J. L. Lebowitz (Academic, New York, 1983).

[19] A.Y. Grossberg and A.R. Khokhlov, Statistical Physics of Maromolecules (AIP Press, New York, 1994), Chapter 7.

[20] In contrast to the finite kink energy, the entropy of an isolated kink diverges logarithmically with system size. See L.D. Landau and E.M. Lifshitz, Statistical Physics (Pergamon Press, Oxford, 1988), Sec. 163.

[21] M.E. Fisher, J. Chem. Phys. 45, 1469 (1966).

[22] Y. Kafri, D. Mukamel, and L. Peliti, Phys. Rev. Lett. 85, 4988 (2000); Eur. Phys. J. B27, 132 (2002).

[23] Y.L. Lyubchenko, A.V. Volodskii, and M. Frank-Kamenetskii, Nature 271, 28 (1978); A.V. Volodskii, A.V. Amrykyan, Y.L. Lyubchenko, and M. Mamenetskii, Biomol. Struct. Dyn. 2, 131 (1984).

[24] L.-H. Tang and H. Chate, Phys. Rev. Lett. 86, 830 (2001); see also D. Cule and T. Hwa, Phys. Rev. Lett. 79, 2375 (1997).

[25] M. Doi and S.F. Edwards, The Theory of Polymer Dynamics (Oxford, New York, 1988).

[26] P. Nelson, Biological Physics: Energy, Information Life (W.H. Freeman, New York 2003). 
[27] P.G. deGennes, Rep. Prog. Phys. 32, 187 (1969).

[28] See, e.g., J. Marko and E.D. Siggia, Science 265, 506 (1994) for a discussion of these effects, which are related to the physics of supercoiling.

[29] For an analysis of the closely related problem of a vortex line in a Type II superconductor interacting with a columnar pin, see D.R. Nelson Defects and Geometry in Condensed Matter Physics (Cambridge University Press, Cambridge, 2002), Chapters 7 and 8.

[30] See, e.g., F. Schwabl, Quantum Mechanics (Springer, Berlin, 1992), Chapter 6.

[31] For potentials like those in Fig. 4, the ground state always remains localized in one and two dimensions provided the well depth $U_{0}>0$. See L.D. Landau and E.M. Lifshitz, Quantum Mechanics (Pergamon Press, New York, 1969).

[32] N. Hatano and D.R. Nelson, Phys. Rev. B56, 8651 (1997), and references therein.

[33] D. Ertas, Phys. Rev. B59, 188 (1999).

[34] J.F. Marko and E.D. Siggia, Macromolecules 28, 8759 (1995).

[35] S.B. Smith, Y. Cui, and C. Bustamante, Science 271, 795 (1996).

[36] S.V. Buldyrev, A.L. Goldberger, S. Havlin, R.N. Mantegna, M.E. Matsa, C.-K. Peng, M. Simons, and H.E. Stanley, Phys. Rev. E51, 5084 (1995).

[37] P. LeDoussal, C. Monthus, and D.S. Fisher, Phys. Rev. E59, 4795 (1999).

[38] Ya. G. Sinai, Theor. Probab. Appl. 27, 247 (1982).

[39] B. Derrida, J. Stat. Phys. 31, 433 (1983).

[40] J.P. Bouchaud, A. Comtet, A. Georges, and P. LeDoussal, Ann. Phys. (N.Y.) 201, 285 (1990).

[41] A.B. Harris, J. Phys. C 7, 1671 (1974). 
[42] C. Danilowicz, V.W. Coljee, C. Bouzignes, D.K. Lubensky, D.R. Nelson, and M. Prentiss. Proc. Nat'l. Acad. Science 100, 1694 (2003).

[43] J. Santa Lucia, H.T. Alawi and P.A. Seneviratne, Biochemistry 35, 3555 (1996).

[44] B. Maier, D. Bensimon and V. Croquette, PNAS 97, 12002 (2000).

[45] U. Gerland, R. Bundschuh and T. Hwa, Biophys. J. 81, 1324 (2001). 